\title{
A Study of the Development of Generative Grammar from the Perspective of Changes in the Movement Pattern
}

\author{
Haojie Li \\ School of Foreign Languages, Southwest University of Political Science and Law, Chongqing, China; \\ Faculty of English Language and Culture, Guangdong University of Foreign Studies, Guangzhou, China \\ Guicheng Wang \\ Xiyou Middle School, Laizhou, Shandong, China
}

\begin{abstract}
Movement plays an important role in generative grammar. This paper expounds characteristics and motivation of the development of generative grammar theory by analyzing and contrasting the movement pattern in different periods of generative grammar. From Move- $\alpha$ to the feature checking, and then to the matching of the probe and the target under agreement feature, a series of changes in movement pattern indicate that generative grammar is exploring the principled interpretation of the language from the perspective of biolinguistics, the explanation of the characteristics and general principles of the interface system, ultimately to the goal of going beyond the explanatory adequacy.
\end{abstract}

Index Terms - movement pattern, generative grammar, Move- $\alpha$, feature checking, GB Theory, MP

\section{INTRODUCTION}

Since generative grammar was founded, every development has involved changes in the theoretical framework and specific analytical techniques. Movement is a case in point and it plays an important role in generative grammar. By analyzing the movement pattern in different periods of generative grammar, we can trace the trajectory of generative grammar development, explore the characteristics of generative grammar development and its motivation, and look forward to the future development direction of generative grammar.

\section{MOVEMENT IN GB THeORY}

The syntactic derivation model of GB Theory is shown in (1) (Wen, 2002):

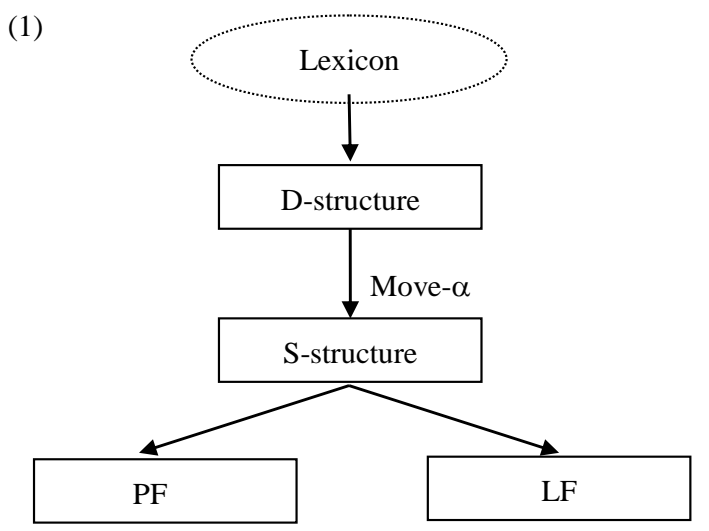

The process of derivation from D-structure to S-structure is called transformation. In its subsequent development, the word "transformation" is gradually replaced by "move", which is now the reason why the transformational generative grammar is called generative grammar. In GB theory, Chomsky $(1978,1980)$ used one rule-Move- $\alpha$ to decompose and merge all kinds of transformational rules in the early stage of the transformational generative grammar, such as Pronominalization, Passivization, Relativization, Equi-Deletion and Dative Movement. The intention of Move- $\alpha$ is to say that any component can participate in the movement, any pattern can be used as a means of movement, any structural position can be the destination of the movement. But this rule does not actually mean that any component can be moved to any position, rather the movement must be limited by various conditions. There are two types of movement: 
NP movement and wh-movement.

\section{A. NP Movement}

We take the passive sentence as an example to investigate the derivation process of NP movement.

Passive sentences are generally believed to be derived from active sentences. For example:

(2) a. The man murdered the woman.

agent patient

b. The woman was murdered.

patient

We can use (2b) as the S-structure, which is transformed from the D-structure, which is represented by (3) by "Move- $\alpha$ ".

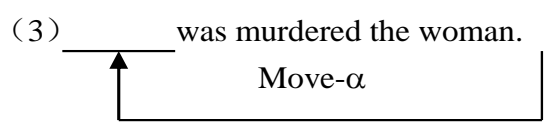

In (3), the moved object is the noun phrase "the woman", and the landing site is the blank subject position. Let us take a closer look at the derivation process of the passive sentence. $(4 b)=(2 b)$ is derived from the D-structure in $(4 a)$.

(4) a. [IP $e$ was murdered the woman].

b. The woman was murdered.

The $e$ of (4a) represents that the subject position in the D-structure is an empty position. According to the theta criterion, the verb murder is used as a two-place predicate, which has two theta roles to be assigned. In (4a), the patient role is assigned to the woman, but because the subject position in the D-structure is an empty position, there is no external argument to assume the role of agent. However (4b) is a grammatical sentence, showing that the role of "agent" in (4a) must be assigned in some way, and thus (4b) does not violate theta criterion. According to Jaeggli (1986) and Roberts (1987), the reason why there is no overt element in the D-structure of the passive sentence to assume the role of agent is because the verb of the passive sentence has undergone a morphological change. The passive morphological change of the verb makes the verb lose the ability to assign the external theta role, that is to say, the passive morphological change of the verb absorbs the role of "agent". Since the subject position in (4a) cannot obtain the theta role, an empty subject position indicated by $e$ is left. According to the Extended Projection Principle (EPP), a sentence must have a subject. If the internal argument in (4a) does not move, we can fill in the expletive "it" in the empty subject position, as shown in (5), but the sentence is not grammatical. Therefore, the movement of the internal argument in the D-structure of the passive sentence is the only way out.

(5) It was murdered the woman.

In (5), "the woman" is the complement of the verb. According to Case Theory, the noun phrase at the position of the complement can get the structural Case from the verb. But if "the woman" gets the structural Case in place, there is no need to move. Obviously, after the passive morphological change of "murder", it not only absorbs the role of agent of the subject, but also absorbs the Case of the complement, so that the verb "murder" loses the ability to assign the structural Case to the complement. "The woman" does not get Case in the complement position, it fails to pass the Case Filter, and the whole sentence is ungrammatical. Therefore "the woman" must move to the position where the Case can be obtained in order to pass the Case Filter. (6) shows that the empty subject position just provides such a condition. The inflection I in the sentence can assign a structural Case to the subject position. Then the internal argument "the woman" is moved to the subject position, and the Case is obtained, which satisfies the EPP and passes the Case Filter, thus ensuring that the entire sentence is grammatical. 


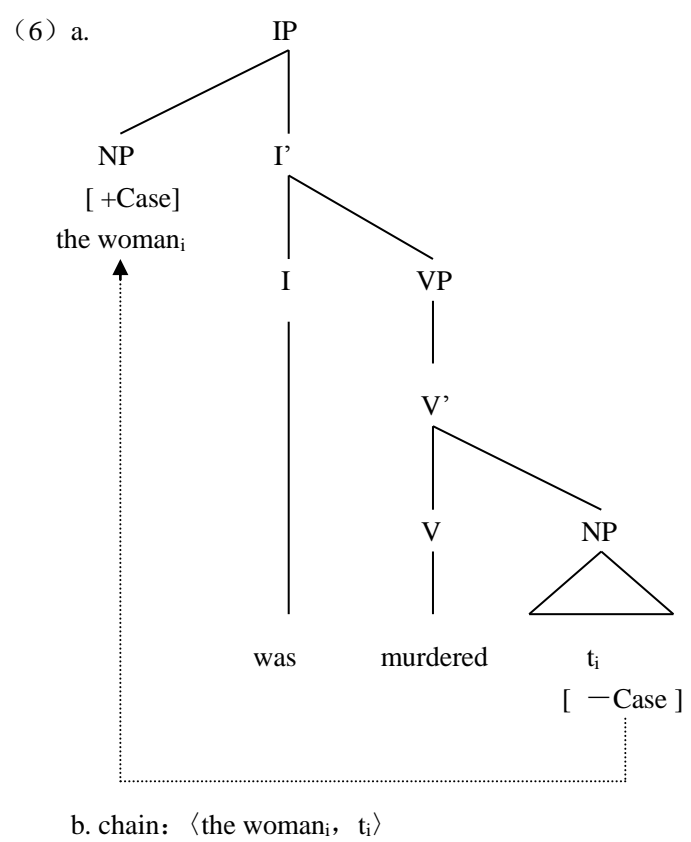

After "the woman" moves to the subject position, it leaves a trace in place, called the trace (represented by $t$ ). The moved element is co-indexed with the trace, forming a chain. In terms of the relationship between the Case and the theta role, it can be standardized by the Visibility Condition: noun phrases can get $\theta$-role on condition that they have Case. In (4a), due to the change of passive form, the verb loses the ability to assign a Case to a complement, and "the woman" does not get a Case in the D-structure. According to the Visibility Condition, the noun phrase cannot obtain $\theta$-role in the D-structure. In the S-structure (4b), "the woman" gets the Case, but at this time the woman is outside the governing category of the verb "murder", and the $\theta$-role should not be obtained. However, (4b) is grammatical. The concept of the chain helps to solve the above problems. The elements of the chain have an inseparable connection and are an integral whole. As long as an element has a Case in the chain, the whole chain is regarded as a visible position; the $\theta$-role is not directly assigned to a certain theta position in the chain, but is assigned to the entire chain. In (6), the chain $\left\langle\right.$ the woman $\left.n_{i}, t_{i}\right\rangle$ the head of the chain-"the woman" gets the Case, so the whole chain becomes a visible position. The verb "murder" assigns the internal theta role to the entire chain. "The woman" at the beginning of the chain obtains $\theta$-role through the chain. Therefore, (4b) conforms to the Visibility Condition, and the entire sentence is grammatical.

\section{B. Wh-movement}

In regard to wh-movement, Subjacency Condition specifies the limit imposed by the wh-movement on the movement distance, and Empty Category Principle limits traces left after wh-movement. Let us take (7) as an example to analyze the case where Move- $\alpha$ is restricted during wh-movement.

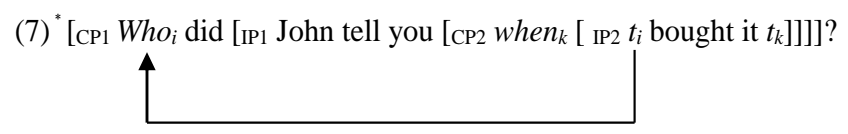

The reason why (7) is ungrammatical is that the wh-word "who" moves from the position $t$ where is located in the D-structure to the beginning of the sentence, and crosses two "IPs". Because the position of the first "IP" is occupied by "when", CP2 constitutes a wh-island, causing "who" to cross two "IP" nodes, violating Subjacency Condition, so the sentence is not grammatical. The trace $t_{i}$ in (7) is in the subject position of IP2, and cannot be theta-governed. It can only rely on the antecedent-government to obtain proper government. The nearest governor is the when $\mathrm{k}_{\mathrm{k}}$ at [Spec, $\left.\mathrm{CP} 2\right]$, but because of $w_{h} n_{k}$ does not co-indexed with ti and it cannot be the antecedent to properly govern ti. Who $\mathrm{i}_{\mathrm{i}}$ is the antecedent of $t_{i}$, but because "When" is the possible governor of $t_{i}$ between $W o_{i}$ and $t_{i}$, Who' ${ }_{i}$ 's proper government of $t_{i}$ cannot comply with minimality, so $t_{i}$ is not subject to any proper government and violates ECP.

In summary, the basic assumption of movement in GB theory is strict entry and tolerant exit, and Chomsky attributes many of the various transformational rules in the early transformational-generative grammar to Move- $\alpha$, but this does not mean any element can be moved to any position, but movement must be limited by various conditions, such as theta criterion, Case Filter, Visibility Condition, Subjacency Condition, Empty Category Principle, etc. to eliminate the ungrammatical structure. However, GB simply lays emphasis on the result and regardless of the movement process will 
result in some syntactic elements are eventually eliminated by other theoretical modules after movement, leading to a half-effect. Then the generative grammar repositions movement in MP. The movement of MP is quite different from the movement of GB in terms of concept and operation.

\section{MOVEMENT IN MP FRAMEWORK}

The grammatical model of MP is shown in (8) (Xu, 2009):

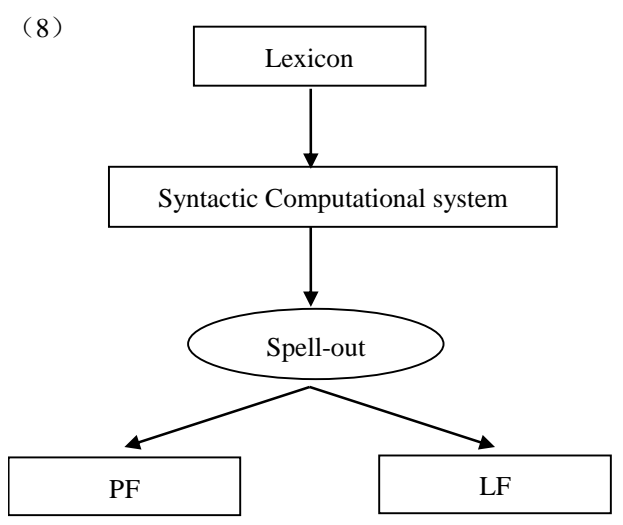

The grammatical model of MP simplifies the production process based on the grammatical model of GB. The grammar model in MP shows that the language consists of two components: lexicon and syntactic computational system, D-structure and S-structure are eliminated, and PF and LF are preserved. In the MP framework, movement becomes internal merge and becomes a means of merging. Movement has evolved from the use of feature checking as a motivation to the use of feature-driven motivation in MP.

\section{A. Movement Motivated by Feature Checking}

During MP, Move- $\alpha$ is removed, claiming that movement is an internal merge (Chomsky 2004). The movement of the initial stage of MP is based on the feature checking. All morphological features in the lexicon, such as $\Phi$ features (person, gender, and number) and Case features, must be checked by some functional category at appropriate positions after entering the sentence. The derivation that passes the checking of a functional category can "converge" to the interface. Feature checking mainly involves semantic uninterpretable features that are deleted after checking and eventually eliminated. The singular and plural characteristics of nouns in English belong to the semantic uninterpretable features and need to be checked. The singular and plural characteristics of English verbs can check the singular and plural characteristics of nouns, as shown in example (9):

(9) The man likes serious music.

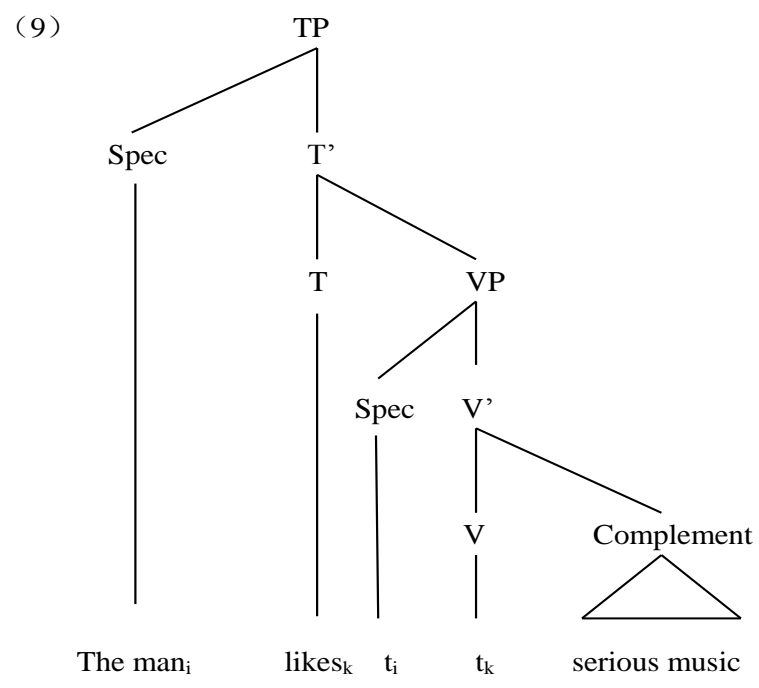

In (9), "the man" at [Spec, VP] position has a semantic uninterpretable singular feature. "Likes" moves to "T" to form [T, likes], which has the ability to check the semantic uninterpretable singular feature. So "the man" must be merged with [T, likes] to the [Spec, TP] position, accepting the checking of [T, likes]. In the end, the singular third-person semantic uninterpretable singular feature of "the man" and the singular third-person semantics of the verb 
"likes" are mutually checked, satisfying the requirements of the checking theory.

\section{B. Movement of Feature-driven Motivation}

Later movement becomes a feature-driven one, and the agreement relationship established between probe and goal plays an important role. Agreement relationship is the consistent relationship between entity category and functional category in formal features and its role in operation. The operation of movement is a combination of merge and agreement (Wu, 2006). Take (10) for example.

(10) We have read that book.

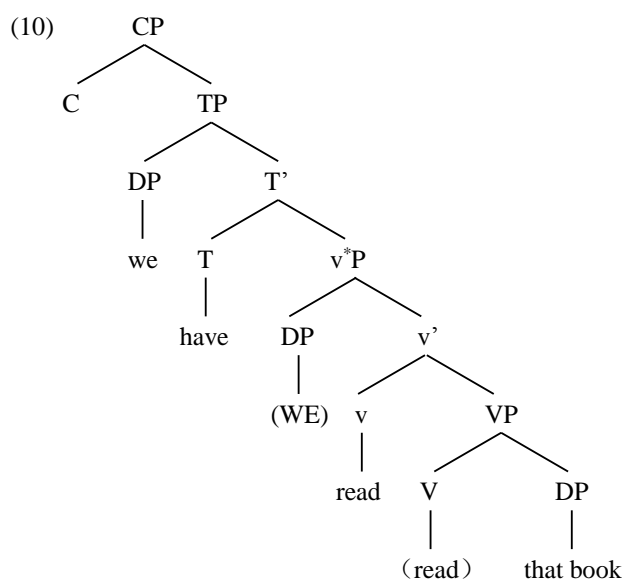

The basic structure of a sentence is $\left[\mathrm{CP}>\mathrm{TP}>\mathrm{v}^{*} \mathrm{P}>\mathrm{VP}\right]$, and $\mathrm{CP}$ and $\mathrm{v}^{*} \mathrm{P}$ are phases (Chomsky 2004). All syntactic operations are determined by the head of phase: $\mathrm{C}$ and $\mathrm{v}^{*}$, and the syntactic derivation is performed by phase. There are two phases in (10): $\mathrm{CP}$ and $v^{*} \mathrm{P}$. The derivation of the structure begins with VP. Lexical items participating in the operation are first extracted from the lexicon to form a lexical array \{we, have, read, that, book , and the lexical array contains the lexical items required for a sentence derivation. Then "read" and "that book" form a lexical sub-array \{read, that book\}, and the lexical sub-array contains only necessary lexical items for one phase derivation. Then "read" and "that book" are merged to form a new syntax object VP. VP then merges with the core functional category $\mathrm{v}$ to form a $v^{*} \mathrm{P} . \mathrm{v}^{*} \mathrm{P}$ has the external argument structure and a complete agreement feature. According to the Feature Inheritance Hypothesis proposed by Chomsky (2007): the inheritance of agreement features is the common feature of the phase heads, $\mathrm{C}$ conveys the agreement feature to $\mathrm{T}$, and the phase head $\mathrm{v}^{*}$ is also transmitted agreement feature to $\mathrm{V}$. After the light verb $v$ and VP are merged, the agreement feature ( $\Phi$-feature) of $v$ is passed to $\mathrm{V}$, so that $\mathrm{V}$ has the ability to assign an accusative Case. The accusative Case of "that book" is assigned, taking on the role of patient of V. Chomsky (2007) assumes that any type of notional verb is moved to the position of the light verb $v$, so that V "read" moves up to the position of $v$. In (10), the elements in parentheses indicate the copies remained in situ and the merged copy forms a chain; after the phase operation is completed and spell-out is performed, the elements in parentheses often do not have phonetic realization. Then DP "WE" and $v^{\prime}$ are merged to form a phase $v{ }^{*} \mathrm{P}$, and DP "WE" assumes the agent role of $\mathrm{V}$. After the $\mathrm{V}^{*} \mathrm{P}$ phase is completed, the complement VP of $v$ is transferred to the phonetic and semantic parts to process further, and VP and its contained elements are no longer involved in the operation of the next phase. It is not detected by the probe in the next phase, which is the Phase Impenetrability Condition (PIC) proposed by Chomsky (2004).

The derivation proceeds, $\mathrm{T}$ merges with $v \mathrm{P}$, and then merges with $\mathrm{C}$, and $\mathrm{C}$ passes the agreement feature to $\mathrm{T}$. $\mathrm{T}$ has tense feature (present tense), agreement feature ( $\Phi$-feature), and EPP feature. The agreement feature of $\mathrm{T}$ and the Case feature of WE are semantically uninterpretable features, so both $\mathrm{T}$ and WE are active. The semantic uninterpretable agreement feature of $\mathrm{T}$ is used as a probe to detect WE in the Spec-v ${ }^{*} \mathrm{P}$ position. The agreement feature of WE is semantically interpretable, and $\mathrm{T}$ and WE establish an agreement relationship, so that their semantic features are valued. The agreement feature of $\mathrm{T}$ is valued with the plural first person, the final form of the item at $\mathrm{T}$ is "have", not "has" or "had", because the tense feature of $\mathrm{T}$ is present tense, the agreement feature is plural first person. WE gets the nominative Case from T, and determines that the final form of the item is "we", not "us" or "our". The EPP feature of T makes "we", which is located at the Spec- $\mathrm{v}^{*} \mathrm{P}$ position to the specifier position of TP, enabling internal merge. After the $\mathrm{CP}$ phase derivation is completed, the complement TP of $\mathrm{C}$ and the rest of the components are handed over to the phonetic and semantic parts, and the derivation of the entire sentence is over.

\section{DisCUSSIONS}

Movement is a major feature of generative grammar. Different periods of generative grammar deal with movement differently. The in-depth study of movement pattern changes can provide a deeper insight into the development and change law of generative grammar and grasp the development direction of the generative grammar in the future. 


\section{A. Beyond Explanatory Adequacy}

Generative grammar always insists that human language is a biological phenomenon. The language system, like other biological systems, is also a natural thing. Chomsky $(2005,2007,2008)$ frequently uses the term "biolinguistics" to emphasize the biological properties of contemporary generative grammar research. Biolinguistics is a cross-disciplinary field. It is a combination of biology and linguistics. It also involves related disciplines such as anthropology, psychology, and neuroscience. Generative grammar has become an important part of biolinguistics. The linguistic view of Chomsky's biolinguistics holds that language faculty is like other organs in the body. It is determined by heredity and can grow, develop and mature in a suitable environment. From Move- $\alpha$ to the feature checking, and then to the matching of the probe and the goal under the agreement feature, a series of movement patterns indicate that the generative grammar is exploring the principled interpretation of the language from the perspective of biolinguistics, exploring the explanation of the characteristics and general principles of the interface system, ultimately to the goal of going beyond explanatory adequacy.

\section{B. Different Movement Patterns between GB and MP}

From the perspective of universal grammar research, the study of GB is mainly to fully explore the role of universal grammar in language acquisition, and thus many principles and parameters are proposed. The research focus of MP is that how small scope the universal grammar can be limited to. The goal of MP is to simplify the linguistic theory, guided by the "economy principle", and the syntax derivation reduces unnecessary expression levels and derivation steps. The generative grammar takes the semantic uninterpretable agreement features as the motivation of movement. When the language is used by the external system, the semantic uninterpretable feature is eliminated, and the interface condition (IC) is satisfied, so that the SM and CI systems respectively obtain effective sound and meaning information to ensure that the syntax operation is successfully derived.

\section{Imperfection of Language Design}

Chomsky pointed out that "displacement" is the ubiquitous phenomenon in human language. It is considered to be the expression of language imperfection, and language has its own way to deal with this seemingly imperfect phenomenon. Movement motivated by features is the way to solve this problem. Therefore, the language design may be perfect from the perspective of getting along between language and other human brain cognitive organs.

\section{Internal Merge and Language Design}

In MP, movement becomes "internal merge" and a way of "merging". "Merge" is a universal operation of human language, "costless" (Chomsky 1995: 226), which can be used repeatedly and at no cost. Hauser, Chomsky, Fitch (2002) published an academic paper on language evolution "The faculty of language: what is it, who has it, and how did it evolve?" and Chomsky believes that "merge" may occur on biological individuals. The result of genetic mutation is the original source of human language. Chomsky believes that "merging" should have a corresponding expression on its genetics. Therefore, movement in MP is internal merge, It is the exploration of genetic inheritance, which is one of the elements of language design from the perspective of biolinguistics.

\section{E. Reflection of Functional Categories}

The development of generative grammar reflects people's deep understanding of the differences in language structure. On the research platform of MP, movement must have a motivation. From the initial attributing language structure differences to verb movement, and the movement of nouns in order to obtain the Case, to the concept of the core functional category in the latest framework, it shows that the grammar can reflect people's understanding of language into the model of syntactic operation in time. The structural differences between languages are mainly in the functional categories.

\section{F. Latest Advances in Movement Patterns}

The generative grammar focuses on the theoretical interpretation of linguistic phenomena, while the traditional grammar focuses on description. If we only satisfy the description and induction of the superficial differences in language, we will not be able to further understand the facts reflected in the language. From the unconstrained Move- $\alpha$; to the movement by the feature checking motivation, the purpose of movement is to delete and finally eliminate the semantic uninterpretable features, and finally to ensure the successful derivation of the syntactic derivation; then to the agreement $\mathrm{f}$ of phase theory: the matching of the probe and the goal, the agreement semantic uninterpretable features of the phase head ( $\Phi$-feature) plays a central role in the syntactic derivation, making the probe in an active state. It is the driving force of the operation, and also the motivation of movement. Latest advances in movement patterns have demonstrated that generative grammar focuses on theoretical interpretation of linguistic phenomena. 


\section{ACKNOWLEDGEMENTS}

This research is funded by General Project of 2017 Chongqing Municipal Education Commission Humanities and Social Sciences Research, P.R.C. Project Name: A Generative Study on the Derivation of the Oblique Object Construction in Mandarin Chinese under the Framework of Distributed Morphology (No. 17SKG003) and 2017 Key Project of Southwest University of Political Science and Law, P.R.C. Project Name: A Syntactic Study on the Verb-copying Construction in Mandarin Chinese (No. 2017XZZD-09).

\section{REFERENCES}

[1] Chomsky, N. (1978). Rules and Representations. New York: Columbia University Press.

[2] Chomsky, N. (1980). On Binding. Linguistic Inquiry 11:1-46.

[3] Chomsky, N. (1995). The Minimalist Program. Cambridge, Mass.: MIT Press.

[4] Chomsky, N. (2004). Beyond explanatory adequacy. In A. Belletti (eds.). Structures and Beyond: The Cartography of Syntactic Structures, 104-131. Oxford: Oxford University Press.

[5] Chomsky, N. (2005). Three factors in language design. Linguistic Inquiry, (36): 1-22.

[6] Chomsky, N. (2007). Approaching UG from below. In U.Sauerland.\& H-M. Gärtner (eds.) Interfaces+Recursion=Language? Chomsky's Minimalism and the View from Syntax-Semantics, 1-29. Berlin: Mouton de Gruyter.

[7] Chomsky, N. (2008). The Biolinguistic Program: Where Does It Stand Today? Keynote speech delivered at the Annual Meeting of the International Association of Chinese Linguistics (IACL), Peking University, Beijing.

[8] Hauser, M. D., N. Chomsky \& W. T. Fitch. (2002). The faculty of language: what is it, who has it, and how did it evolve? Science 298, 1569-1579.

[9] Jaeggli, O. (1986). Passive. Linguistic Inquiry, 17:587-633.

[10] Roberts, I. (1987). The Representation of Implicit and Dethematized Subjects. Dordrecht: Foris.

[11] Wen, B. L. (2002). An introduction to syntax. Beijing: Foreign Language Teaching and Research Press.

[12] Wu, G. (2006). A Study on Generative Grammar. Shanghai: Shanghai Foreign Language Education Press.

[13] Xu, L. J. (2009). Theories of Generative Grammar: from Standard Theory to Minimalism. Shanghai: Shanghai Education Press.

Haojie Li was born in Shandong, China in 1974. He received his M.A. degree in Sichuan International Studies University in 2005 and he is a PhD candidate majored in English Language and Literature at Guangdong University of Foreign Studies.

$\mathrm{He}$ is currently a lecturer in the School of Foreign Languages, Southwest University of Political Science and Law, Chongqing, China. His research interests include syntax and theoretical linguistics. He has published a number of studies in his areas of expertise, which have appeared in scholarly publications in China and Europe.

Guicheng Wang was born in Yantai, Shandong, China in 1969. He is majored in English teaching and now the vice principal of Xiyou Middle School. 